\section{Immunisation status of dental practice staff in Kent}

\author{
A. Rhodes, ${ }^{1}$ T.-C. Aw, ${ }_{1}^{2}$ C. Allen ${ }^{3}$ and M. Ridout ${ }^{4}$
}

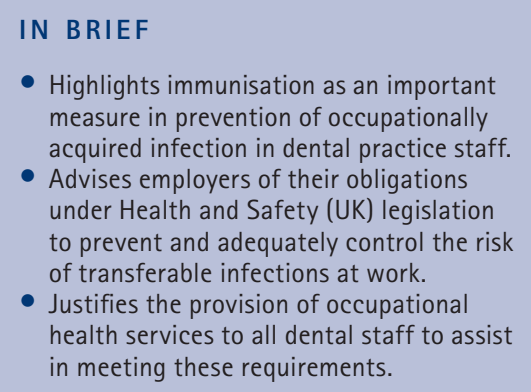

Aim To determine the hepatitis $B$, tuberculosis (TB), varicella and rubella immunisation status of dental practice workers in Kent. Method A cross-sectional survey using a) a dental practice questionnaire sent to all 275 registered dental practices in Kent in February 2005, to determine the numbers of staff employed and their job titles, and b) a confidential personal health questionnaire for every staff member employed by each practice, to determine past history of infections and immunisation history. Results Two hundred out of 257 (78\%) dental practices took part in the survey, and 1,415 staff (76\% of known participants) returned completed personal health questionnaires. Three hundred and eighty-four out of 395 dentists (97\%) indicated previous immunisation against hepatitis B. The corresponding percentages for other occupational groups were dental hygienists (94\%), nurses (89\%), dental therapists (75\%), and other non-clinical staff (65\%). 1,197 (85\%) of participants reported previous chicken pox and/or shingles; 1,208 (85\%) gave a history of previous immunisation against TB; and 823 (58\%) had either had rubella or were immunised against rubella. Male participants were less likely to have had rubella immunisation. Conclusions The study has demonstrated the variations in knowledge about personal immunity status amongst dental practice staff for some infectious diseases. Improvement in establishing personal immunity status of individual dental care workers and provision of a vaccination programme could be facilitated. This preventive measure could be arranged through occupational health providers.

\section{INTRODUCTION}

Current advice from the Department of Health recommends that all workers who have direct contact with blood and other potentially infectious body fluids should be immunised against hepatitis B. ${ }^{1}$ Dental practice staff other than clinical workers, such as receptionists and managers, may require immunisation if involved with disinfection or sterilisation of equipment and disposal of clinical waste. The

\footnotetext{
${ }^{1}$ Specialist Registrar in Occupational Medicine, Health Management Ltd, Ash House, The Broyle, Ringmer, East Sussex, BN8 5NW; ${ }^{2}$ Professor and Head of Department, Department of Community Medicine, Faculty of Medicine and Health Service, United Arab Emirates University,

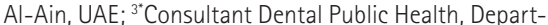
ment of Dental Public Health, Medway PCT, New Court, 1 New Road, Rochester, Kent, ME1 1BD; ${ }^{4}$ Reader in Statistics, Institute of Mathematics, Statistics and Actuarial Science, University of Kent, Canterbury, CT2 7NF

${ }^{*}$ Correspondence to: Christopher Allen

Email: christopher.allen@nhs.net
}

\section{Online article number E20}

Refereed Paper - accepted 30 May 2008

DOI: 10.1038/sj.bdj.2008.999

${ }^{\circledR}$ British Dental Journal 2008; 205: E20 immunity/carrier status of staff performing exposure prone procedures should be determined to protect patients against the risk of acquired hepatitis B from an infected healthcare worker. ${ }^{2}$ Access to an occupational health service capable of providing advice and assistance in immunisation and producing appropriate documentation would be beneficial, so fulfilling the employers' obligations under health and safety legislation, as well as complying with the new GDC registration procedures. In 2001 the UK committed itself to supporting an occupational health service for all healthcare workers ${ }^{3}$ and by 2003 allocated funding through primary care trusts to extend occupational health services to dental practitioners and their staff. ${ }^{4}$

The Control of Substances Hazardous to Health Regulations (COSHH 2002) require employers to consider measures to reduce risks to employees from all workplace biological hazards, including infections. ${ }^{5}$ The Department of
Health now recommends all healthcare workers in general practice, including dental practice staff, to be immunised against a range of preventable occupationally acquired infections including hepatitis B, tuberculosis (TB), varicella and rubella. ${ }^{2}$

Despite these recommendations, the provision of an immunisation service for dental practice staff is still patchy in the UK. ${ }^{4}$ A starting point before beginning immunisation programmes for any occupational group is to determine the magnitude of the task. For dentists and dental practice staff, this would require an estimate of the numbers requiring immunisation, either because the individuals have not previously acquired natural immunity or because they have not been vaccinated before. The approach used in this study was to use questionnaires to obtain this information. This was administered as part of a cross-sectional survey of dental practices carried out in February 2005 across Kent. 
Table 1 Response rates to practice and personal questionnaires and hepatitis B immunisation status of staff in dental practices in Kent

\begin{tabular}{|c|c|c|c|c|c|c|c|c|c|c|c|}
\hline & \multicolumn{5}{|c|}{ CLINICAL STAFF } & \multicolumn{6}{|c|}{ NON-CLINICAL STAFF } \\
\hline & Dentists & Nurses & Hygienists & $\begin{array}{l}\text { Dental } \\
\text { Therapists }\end{array}$ & Total & $\begin{array}{l}\text { Dental } \\
\text { Technicians }\end{array}$ & $\begin{array}{l}\text { Practice } \\
\text { Managers }\end{array}$ & Receptionists & $\begin{array}{l}\text { Domestic } \\
\text { workers }\end{array}$ & Others & Total \\
\hline $\begin{array}{l}\text { Number recorded } \\
\text { on practice } \\
\text { questionnaire }\end{array}$ & 524 & 653 & 125 & 12 & 1,314 & 16 & 81 & 335 & 99 & 23 & 554 \\
\hline $\begin{array}{l}\text { Number } \\
\text { returning } \\
\text { personal } \\
\text { questionnaire }\end{array}$ & 395 & 567 & 123 & 12 & 1,097 & 7 & 81 & 204 & 18 & 8 & 318 \\
\hline $\begin{array}{l}\text { Percentage } \\
\text { of total }\end{array}$ & 75 & 87 & 98 & 100 & 83 & 44 & 100 & 61 & 18 & 35 & 57 \\
\hline $\begin{array}{l}\text { Number } \\
\text { immunised } \\
\text { against } \\
\text { hepatitis B }\end{array}$ & 384 & 507 & 116 & 9 & 1,016 & 3 & 71 & 121 & 6 & 5 & 206 \\
\hline $\begin{array}{l}\text { Percentage } \\
\text { immunised }\end{array}$ & 97 & 89 & 94 & 75 & 93 & 43 & 88 & 59 & 33 & 63 & 65 \\
\hline
\end{tabular}

\section{METHODS}

The cross-sectional survey consisted of the administration of two separate questionnaires:

a) A postal questionnaire seeking information on each dental practice was sent to all registered dental practices throughout Kent. The addresses of the practices were identified from the general dental practice lists held by the primary care trusts (PCTs) in the area in February 2005. An accompanying letter addressed to the principal dentist at each practice explained the purpose of the study. This 'practice questionnaire' was used to identify the number of employees, their job titles (ie dentists, dental nurses, dental hygienists, dental technicians, dental therapists, receptionists, domestic workers and others), and whether they were full-time or part-time, for each member of staff

b) Copies of a second self-administered questionnaire requiring personal data (ie age, sex and confirmation of specific job within the practice) were provided to each practice for every member of staff. Each member of staff was requested to complete this confidential 'personal questionnaire.' Information was also sought on previous infection/immunisation for hepatitis B, varicella and herpes zoster, TB and rubella. In regards to hepatitis B, questions were also

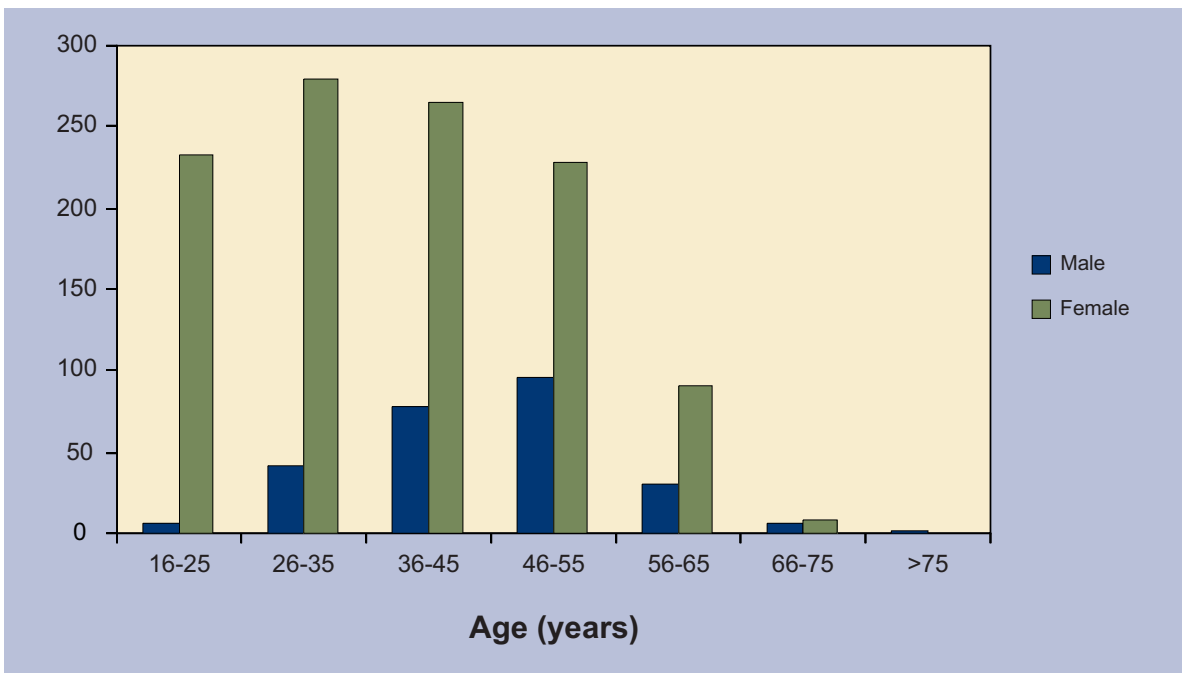

Fig. 1 Age and sex profile of participants from dental practices in Kent

asked regarding booster doses of vaccine received, whether there was an antibody level check and if there was a confirmatory paper record on where and when vaccination was performed.

Pilot testing of the questionnaires involved 15 dental practices outside the study area. Minor modifications were made following pilot testing and the final versions distributed to the practices. Completed personal questionnaires were returned in sealed envelopes to the practice, to be forwarded together with the practice questionnaire to the Department of Dental Public Health in Rochester. A reminder letter was sent after four weeks to those practices that had not replied. Information from each practice questionnaire was collated and the records of each personal questionnaire entered onto a password-protected database. Personal questionnaires with incomplete information were excluded from the study.

Ethical clearance for the study was obtained through the East Kent Hospitals NHS Trust Local Research Ethics Committee.

\section{RESULTS}

In February 2005 there were 257 dental practices registered with the Kent PCTs. The Dental Register for Kent showed 680 registered dentists at that time. The principal dentists from 200 of these practices returned the completed 'practice questionnaire' (a response rate from dental practices of 78\%). There were 524 
dentists (including non-principals) altogether in the 200 practices. There was no indication that non-responders tended to be from a particular geographical area, or associated with smaller sized practices. All practices returning the questionnaire had a practice manager amongst their staff and this may have contributed to a greater participation rate from such practices. The 'practice questionnaire' indicated a total of 1,868 possible participants in nine different occupational categories (Table 1).

1,415 'personal questionnaires' were returned out of a possible total of 1,868. Seven questionnaires had incomplete information, and were therefore removed from the study. The response rate for this questionnaire was 76\%. Figure 1 shows the age and sex profile of staff returning the completed 'personal questionnaires'. The participation rates (Table 1) for practice managers and clinical staff tended to be better ( $75 \%$ or more) than the dental practice staff in other job categories (61\% or less).

\section{(a) Hepatitis B}

Three hundred and ninety-five dentists (75\%) returned the personal questionnaire, of whom 384 (97\% of those returning the questionnaire) reported having been immunised against hepatitis B. Seven hundred (57\%) out of 1,222 total respondents who reported having been immunised against hepatitis $B$ received the vaccination more than five years ago. Of these, 517 (74\%) recalled having had a subsequent booster dose of vaccine, of whom 350 could recollect the year when it was given; 502 (72\%) remembered having had an antibody level check but only 375 (54\%) recalled having been provided with the results. Of those who had been immunised against hepatitis B, 306 (25\%) staff recalled being charged a vaccination fee, $962(79 \%)$ received the immunisation course from their general practitioners, and the remainder from a variety of other sources. Only 307 (25\%) dental practice staff that had been immunised had a paper record to document their hepatitis B immunisation status. A smaller number still were due to have an antibody level determination after completing the full course of hepatitis B immunisation. Other studies of hepatitis
B immunisation have shown that general practice and dental healthcare staff were unlikely to have had their antibody levels checked after vaccination. ${ }^{6,7}$

\section{(b) Other infections}

1,204 of 1,415 respondents (85\%) gave a history of chicken pox and/or shingles and 1,205 (85\%) indicated previous immunisation against TB. Eight hundred and twenty-three of 1,415 (58\%) respondents reported having been immunised against rubella and $536(38 \%)$ recalled previous rubella infection. Older dental practice staff were less likely to have been immunised (Fig. 2), and males were also less likely to have been immunised against rubella (Table 2). The difference between male and female participants in terms of rubella immunisation was statistically significant (Chi-squared test, $1 \mathrm{df}, \mathrm{p}<0.001$ ).

\section{DISCUSSION}

Several Department of Health recommendations, guidance notes and legislation have led to an increasing need to consider immunisations against various infections as part of the provision of occupational health services to healthcare staff.
Guidance on hepatitis B was issued in 1993 requiring all staff primarily performing exposure prone procedures to be immunised against hepatitis $B$ and to have their serological response checked. ${ }^{1}$ In this study, 97\% of dentists and $89 \%$ of dental nurses indicated that they were immune to hepatitis B. These results are comparable to a previously reported study of dental practitioners in 1993. ${ }^{8}$ It is against GDC regulations to practice as a dentist without evidence of immunisation for hepatitis B from November 2006. The GDC is encouraging applications for registration from all dental care professionals by providing a healthcare certificate confirming immunisation. Registration for dental nurses and technicians became compulsory on 31 July $2008 .^{9}$

Sixty-five percent of non-clinical staff in general dental practice had received hepatitis B vaccination. It is not known whether this was a result of their current employment in a dental practice, previous employment in a clinical setting, or as part of their travel abroad. The majority of the participants received vaccination more than five years ago, but few could recall if they received a booster

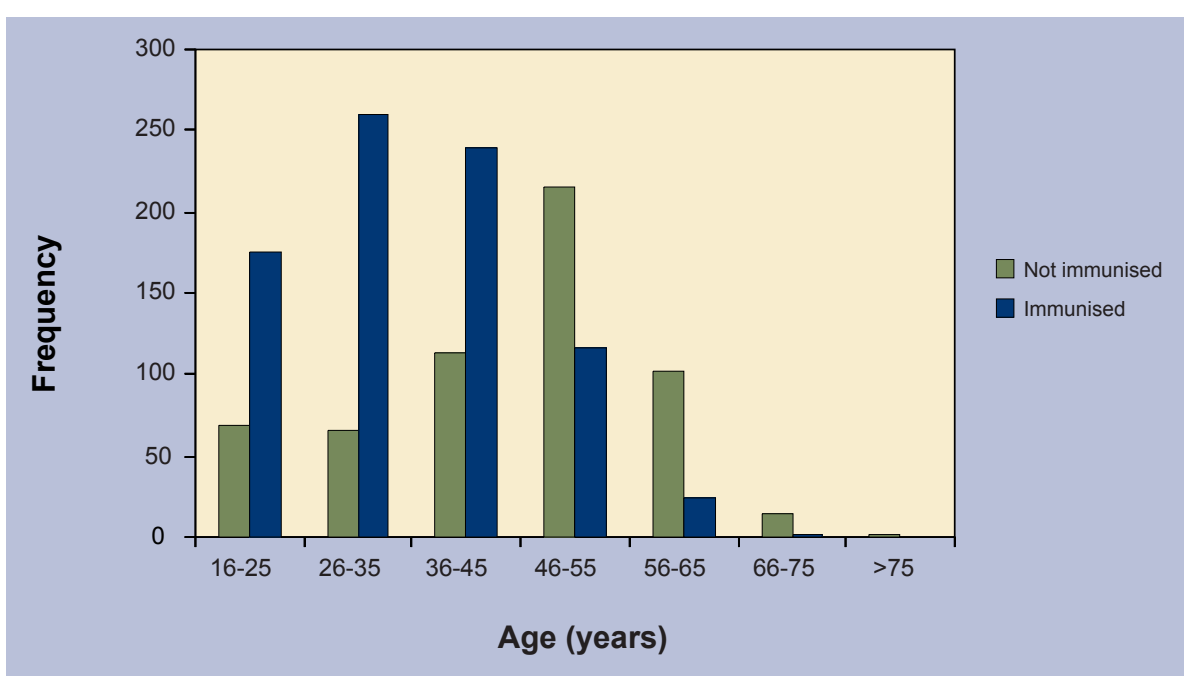

Fig. 2 Number of workers immunised against rubella, by age

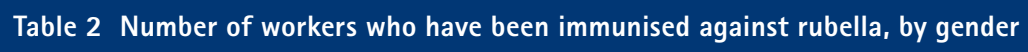

\begin{tabular}{l|l|l|l|}
\hline Gender & Immunised & Not immunised & Total \\
\hline Male & $81(30 \%)$ & $189(70 \%)$ & 270 \\
\hline Female & $723(65 \%)$ & $392(35 \%)$ & 1,115 \\
\hline Total & $804(58 \%)$ & $581(42 \%)$ & 1,385 \\
\hline
\end{tabular}


dose of vaccine or even the year when this occurred. Only a minority had a paper record of their vaccination history.

A logistical issue in regards to the provision of occupational health for dental staff will be to consider whether to establish immunity status by checking antibody (and antigen) status before offering vaccination, or to immunise those with uncertain status at the same time as determining their serological status. The implications of testing positive for antigen status (if this was done) are considerable. It is unlikely that a dental clinician who is surface-antigen positive would be allowed to continue clinical dental practice. All UK dental schools should now provide information to prospective candidates on the need for health clearance prior to the commencement of training to reduce risks to patients and avoid subsequent difficult decisions regarding a career change.

The European Consensus Group published guidelines in 2000 suggesting that repeated booster doses of hepatitis B vaccine may be unnecessary in immunocompetent individuals who have responded adequately to a primary course of vaccine. ${ }^{10}$ The current UK guidelines remain unaltered at recommending a single booster dose five years after the initial full course. ${ }^{11}$

In this study, 395 dentists returned completed personal questionnaires. Eleven of them (3\%) indicated that they have not been immunised against hepatitis B. If the proportion of dentists who have not been immunised against hepatitis B (3\%) is similar among study participants and those who did not participate, then we might expect that 20 dentists in Kent would require hepatitis B immunisation.

The practice questionnaire returns showed a total of 653 dental nurses in 200 dental practices (out of a possible 257 dental practices registered in Kent - 78\%). Five hundred and sixty-seven nurses (87\%) completed the personal questionnaire and 507 of these (89\%) indicated they were immune to hepatitis $\mathrm{B}$. If again it is estimated that the 653 dental nurses come from the 78\% of practices, there could be a possible 837 dental nurses working in Kent practices. If $89 \%$ were immune, this would leave 92 (11\%) requiring immunisation.

The costs involved in immunising these healthcare workers would be the vaccine and equipment (syringes, hypodermic needles), estimated at around $£ 44$ per person at 2006 prices, plus the cost of staff time for vaccine administration. Laboratory charges for determination of immune status would also need to be added. To immunise the possible 92 nurses and 20 dentists would incur an estimated cost of $£ 4,928$ for the vaccine and equipment alone.

Exposure to blood-borne pathogens poses a serious risk to healthcare workers involved in exposure prone procedures. The provision of an immunisation programme would only form one part of a comprehensive occupational health programme of management of risk to exposure to human immunodeficiency virus (HIV), hepatitis B (HBV) and hepatitis C (HCV). Policies and procedures to minimise the risk of occupational bloodborne pathogen transmission, including information and training for dental staff in universal precautions, prompt reporting of needle stick injuries, evaluation, counselling and treatment and followup are necessary, as is active promotion and encouragement to use the available occupational service. A recent study from the United States suggests dental practice staff remain at a high risk of needle stick injury due to the frequent use of syringe needles in local anaesthetic procedures. ${ }^{12}$ Needle stick injury incidents are still likely to be under-reported in the UK. ${ }^{13}$ Although an enhanced surveillance system was launched by the Health Protection Agency Centre for Infections (HPA CfI) in 1997, the availability of information about community dental workers has been limited to those who have access to NHS occupational health services via its 150 participating centres. ${ }^{14}$ The use of the HPA CfI system should be actively encouraged to ensure maximal protection and the availability of optimal medical care for all dental health workers.

Tuberculosis (TB) is re-emerging as a public health problem. The number of cases in England and Wales declined in the 1980s but there has been a recent increase, especially in urban areas and amongst ethnic minority groups from TB endemic areas such as the Indian subcontinent and sub-Saharan Africa. ${ }^{15}$ A recent study showed a twofold increase in TB amongst healthcare workers in England and Wales. ${ }^{16}$ The British Thoracic Society recommends that for healthcare workers, pre-employment and on-employment screening should include obtaining a history of disease or relevant symptoms, previous $\mathrm{BCG}$, and the presence or otherwise of a BCG scar. Tuberculin testing and chest $\mathrm{X}$-rays are to be used only if indicated. ${ }^{17}$ The majority of dental practice workers in this study (85\%) had been previously vaccinated against $\mathrm{TB}$, in part reflecting the success of BCG vaccination in the schools programme introduced in 1953. The remaining 15\% without a previous history of BCG immunisation need to be assessed before vaccination, particularly those who are immigrant workers arriving from countries where a high prevalence of $\mathrm{TB}$ is known to exist. Ensuring that non-immune dental practice staff have no evidence of active disease and provision of an opportunity for BCG vaccination should minimise the risk of TB in staff and potential of spread to others including patients. BCG vaccine is, however, contraindicated in immunocompromised individuals, and this would need to be considered before administering BCG. ${ }^{18}$

Varicella and rubella immunisations are now recommended for all nonimmune healthcare workers in general practice, including those in dental practice. $^{19,20}$ The level of immunity in the study group of dental staff for varicella determined from the personal questionnaire appears similar to the estimated 90\% immunity in the general population. ${ }^{21}$ As there has been a trend towards an increase in infection rate in adults and a higher complication risk, ${ }^{22}$ vaccinating those without a clear previous history of having had chickenpox or shingles after first confirming non-immunity would reduce the incidence of varicella in this occupational group. Protecting all dental workers against varicella will also reduce morbidity and sickness absence from this infection. Chicken pox is not a notifiable disease in England and Wales; most cases are not investigated microbiologically and hence surveillance 
data is not available from the Health Protection Agency.

In this study, $58 \%$ of respondents recalled being immunised against rubella. In the UK, immunisation was first introduced in 1970 to teenage girls and non-immune women of childbearing age. The introduction of the programme reduced the number of cases of CRS and therapeutic abortions. In 1988, the triple vaccination MMR (measles, mumps and rubella) was offered in childhood. Part of this was in the hope of eliminating rubella from the community, as non-immune women were at risk of contracting the infection from children. The number of cases of rubella in children initially fell, although outbreaks occurred in young men in college or university. In the light of advice that all healthcare workers should receive rubella vaccination if not immune, ${ }^{20}$ it is appropriate to consider offering a programme of vaccination to dental practice staff who are in regular contact with pregnant women and children. Male dental workers were significantly more likely not to be immunised against rubella and this may be a result of the policy of selective immunisation of women in the past, or that they perceive that the infection is primarily a problem for pregnant women. Ensuring immunity against rubella for both male and female dental workers is appropriate as it is likely they are in regular contact with pregnant women and children. Reduction of the risk of transmission between dental workers and their patients will further contribute to a reduction in the incidence of disease and possible complications in dental staff and their patients, although rubella is now a very rare infection in the UK.

Questionnaire surveys are subject to participation, information and recall bias. Limitations of this study include dependence on accurate recall, and a possible bias resulting from non-participation. Non-participants could include a larger proportion of individuals who have not been immunised. They may be reluctant to participate because of the perceived implications of declaring their non-immune status. Declaration of non-immunity could lead to a require- ment that they face the inconvenience of arranging immunisation or even discontinuing clinical work. There may also be a greater tendency to indicate that immunisation had occurred if participants believed it would be of benefit to be considered 'safe' and have no restrictions placed on their ability to continue with their practice involving patient care.

A greater proportion of clinical staff compared to non-clinical workers amongst the participants may have contributed to an overestimate of the level of overall immunity. The low participation rate in domestic workers and dental technicians may be due to a belief that the study was not relevant to them, or because they do not perceive any risk of infection since they are not involved in direct patient contact. The length of time in post may be a factor that could have affected the proportion of staff indicating immunisation against hepatitis B, as new staff, eg dental nurses, may have started on a course of immunisation but not completed their full course of immunisation at the time of participation in the study.

\section{CONCLUSIONS}

This study refers to the necessity of setting up an immunisation programme for dental practice staff as part of occupational health services for this group of healthcare workers. The data from this study in Kent, if extrapolated to the rest of the UK, would indicate a large proportion of dental healthcare workers requiring vaccination (a full course and/or booster doses) for several vaccine-preventable infections. Further studies could include an audit and evaluation of the immunisation provisions by an occupational health service in accordance with the Department of Health guidelines. It would be useful to demonstrate whether the levels of immunity for various infections improve and if new cases of these infections in dental staff decline, together with a concomitant reduction in associated sickness absence.

We thank Dr Ranji Kulasegaram for carrying out the pilot study, the staff of the Department of Dental Public Health, Rochester, Kent for administering the study and all the staff of the Kent Dental Practices who participated.
1. Department of Health. Protecting healthcare workers and patients from hepatitis B. Recommendations of the Advisory Group on Hepatitis. London: HMSO, 1993.

2. Department of Health. Health clearance for tuberculosis, hepatitis $B$, hepatitis C and HIV: new healthcare workers. London: HMSO, 2007.

3. Department of Health. The provision of occupational health and safety services for general medical practitioners and their staff. London: HMSO, 2001.

4. Department of Health. Occupational health services for general dental practitioners and their staff. London: HMSO, 2003. NHS Gateway Communication Reference No. 2595.

5. Health and Safety Executive. Control of Substances Hazardous to Health Regulations 2002 (as amended). Approved code of practice and guidance. Norwich: HSE Books, 2005

6. Whittle $J \mathrm{G}$. An audit of the hepatitis B vaccination status of staff in general denta practices in Lancashire. Primary Dent Care 2003: 10: 27-29.

7. Smith N A L. Management of hepatitis B immunisations and blood exposure incidents in primary care. Occup Med 2004; 54: 231-237.

8. Scully C, Griffiths M, Blake C. Control of cross infection in UK clinical dentistry in the 1990s: immunisation against hepatitis B. Br Dent J 1993; 174: 29-31.

9. General Dental Council. GDC simplifies health checks for dental care professionals. London: GDC, 2006. News release, November 10 2006. Available from http://www.gdc-uk.org

10. European Consensus Group on Hepatitis B immunity. Are booster immunisations needed for lifelong hepatitis B immunity? Lancet 2000; 355: 561-565.

11. Department of Health. Immunisation against disease. London: HMSO, 2007.

12. Shah S, Merchant A T, Dosman J A. Percutaneous injuries among dental professionals in Washington State. BMC Public Health 2006; 6: 269-278.

13. Elder A, Paterson C. Sharps injuries in UK health care: a review of injury rates, viral transmission and potential efficiency of safety devices. J Occup Med 2006; 56: 566-574

14. Health Protection Agency. Reporting of occupational exposure to bloodborne viruses - history and how to report webpage. http://www.hpa.org. uk/webw/HPAweb\&HPAwebStandard/HPAweb_C /1195733803345? $\mathrm{p}=1191942146594$ (accessed 7 October 2008).

15. Department of Health. Stopping tuberculosis in England: an action plan from the Chief Medical Officer. London: DOH Publications, 2004.

16. Meredith S, Watson J M, Citron K M et al. Are healthcare workers in England and Wales at increased risk of tuberculosis? BMJ 1996: 313: $522-525$

17. Joint Tuberculosis Committee of the British Thoracic Society. Control and prevention of tuberculosis in the United Kingdom: code of practice 2000. Thorax 2000; 55: 887-901.

18. The National Collaborating Centre for Chronic Conditions. Tuberculosis: clinical diagnosis and management of tuberculosis, and measures for its prevention and control. London: Royal College of Physicians, 2006.

19. Department of Health. Chickenpox (varicella) immunisation for health care workers. London: Department of Health, 2003. NHS Gateway Reference No. 2003.

20. Department of Health. Protecting women against rubella: switch from rubella vaccine to MMR. London: Department of Health, 2003. PL CNO(2003). NHS Gateway Reference No. 2003.

21. Bramley J C, Jones I G. Epidemiology of chicken pox in Scotland: 1981 to 1998. Commun Dis Public Health 2000; 3: 213-215.

22. Joseph C A, Noah N D. Epidemiology of chicken pox in England and Wales 1867-1985. BMJ 1988; 296: 673-676. 